\title{
Highly accurate pose estimation as reference for autonomous vehicles in near-range scenarios
}

\author{
Ursula Kälin'1, Louis Staffa ${ }^{2}$, David Eugen Grimm ${ }^{1, *}$ and Axel Wendt ${ }^{2}$ \\ 1 University of Applied Sciences and Arts Northwestern Switzerland, Institute Geomatics, Muttenz, Switzer- \\ land; ursula.kaelin@fhnw.ch \\ 2 Robert Bosch GmbH, Stuttgart, Germany; Louis.Staffa@de.bosch.com, Axel.Wendt@de.bosch.com \\ * Correspondence: david.grimm@fhnw.ch;
}

\begin{abstract}
To validate the accuracy and reliability of onboard sensors for object detection and localization in driver assistance, as well as autonomous driving applications under realistic conditions (indoors and outdoors), a novel tracking system is presented. This tracking system is developed to determine the position and orientation of a slow-moving vehicle (e.g. car during parking maneuvers), independent of the onboard sensors, during test maneuvers within a reference environment. One requirement is a 6 degree of freedom $(\mathrm{DoF})$ pose with a position uncertainty below $5 \mathrm{~mm}(3 \sigma)$, an orientation uncertainty below $0.3^{\circ}(3 \sigma)$ at a frequency higher than $20 \mathrm{~Hz}$, and a latency smaller than $500 \mathrm{~ms}$. To compare the results from the reference system with the vehicle's onboard system, a synchronization via Precision Time Protocol (PTP) and a system interoperability to Robot Operating System (ROS) is implemented. The developed system combines motion capture cameras mounted in a $360^{\circ}$ panorama view set-up on the vehicle with robotic total stations. A point cloud of the test site serves as a digital twin of the environment, in which the movement of the vehicle is simulated. Results have shown that the fused measurements of these sensors complement each other, so that the accuracy requirements for the $6 \mathrm{DoF}$ pose can be met, while allowing a flexible installation in different environments.
\end{abstract}

Keywords: Motion capture camera, robotic total station, autonomous vehicle, 6 DoF pose estimation, accuracy

\section{Introduction}

Autonomous driving algorithms use different integrated sensors (camera, lidar, radar, ...) whose outputs are fused to provide information for controlling the vehicle. For the safe release of such autonomous vehicles, these sensors must fulfill strict requirements in terms of spatial and detection accuracy. Environmental conditions like weather effects, external light sources and other traffic participants can influence the sensors' performance, potentially impacting behavior in real traffic situations. Therefore, any argument for the release of an autonomous vehicle must be based on data that reflect the conditions in real traffic.

Methods that can be used to achieve sufficient testing of the sensors under the required conditions are statistical analyses of drives in real traffic, as well as scenario-based simulations. These approaches cover most requirements and can be used for release arguments, but they can also be very costly and favor scenarios that occur frequently during regular traffic.

A special set of scenarios that autonomous driving vehicles must handle involve near-range scenarios such as maneuvering in and out of parking spots. These situations usually only occur a few times per drive and at low speeds, but they require a higher accuracy of localization and object detection, compared to flowing traffic. While an autonomous vehicle can keep a safe distance of more than a meter from any traffic participant at any time in regular traffic, a tight parking spot might require maneuvering with only $20 \mathrm{~cm}$ of space or less on either side of the vehicle. 
A novel, independent measuring system is necessary in order to provide proof that the autonomous vehicle's sensors fulfill the necessary requirements in terms of accuracy and reliability in such near-range scenarios of accuracy and reliability in realistic nearrange scenarios. Due to the mostly static environment and low driving speeds while parking indoors and outdoors, typical references such as global navigation satellite system (GNSS) devices on target vehicles and onboard inertial measurement units (IMU) can only be used to a limited extent due to missing satellites reception in indoor scenarios (GNSS) and drift behavior of the IMU. Instead, a novel combination of external sensors to track the precise position and orientation of the vehicle under test in a local reference system, was developed in this research.

The integration of this tracking system into the existing onboard sensor environment of the vehicle leads to additional requirements. This includes the establishment of a link between the reference frames as well as the communication between the components. The accuracy requirements are specified to be one order of magnitude higher than the tested system at a similar message frequency.

Despite the large range of systems for determining positions or poses, there is no standard system that meets all the requirements modeled in this publication. The requirement of a 6 degree of freedom (DoF) pose with a position uncertainty below $5 \mathrm{~mm}(3 \sigma)$, orientation uncertainty below $0.3^{\circ}(3 \sigma)$, at a frequency higher than $20 \mathrm{~Hz}$ and a latency smaller than $500 \mathrm{~ms}$, could be achieved by known methods in industrial metrology. However, the system still needs to work outside of laboratory conditions, which means varying meteorological and illumination conditions as well as a measuring range, which is requested to be of up to $100 \mathrm{~m}$. This section provides an overview of the measurement systems considered.

Robotic total stations or multi stations determine the coordinates of a target (e.g. prism) by measuring horizontal and vertical angles and distances. Thanks to automated target aiming, it is possible to automate the tracking and measuring of a $360^{\circ}$ prism mounted on a vehicle. A Leica MultiStation MS60 has a specified accuracy for angle measurements of $1^{\prime \prime}$ ( 0.3 mgon) and distance measurements of $1 \mathrm{~mm}$ (+ 1.5 ppm) [1]. Nevertheless, the structure of the $360^{\circ}$ prism introduces additional systematic deviations dependent on its orientation. [2] show that for a GRZ122 prism, cyclic errors of more than $1 \mathrm{~mm}$ can be detected, along with larger deviations for specific prism orientations. The limiting factor of the maximum achievable measurement rate of $20 \mathrm{~Hz}$ is the performance of the electronic distance measurement (EDM), as described by [3].

Using the Leica GeoCOM communication interface allows for the integration of the MS60 in a sensor network and the control of the instrument from an external program. However, this interface does not have a native synchronization option based on standardized network-based synchronization protocols. Investigations from $[4,5]$ regarding total stations show that the synchronization quality gains more significance for the spatio-temporal accuracy, the faster the movement of a tracked prism.

In order to estimate the 6 DoF-pose, it is necessary to have simultaneous measurements of three prisms on a vehicle. Assuming possible measurement deviations of $4.3 \mathrm{~mm}$, $5.0 \mathrm{~mm}$ and $2.9 \mathrm{~mm}$ in longitudinal and lateral respective vertical directions, the required baseline length to meet the orientation accuracy can be calculated using a variance propagation with partial derivatives of $\tan ^{-1}\left(\frac{x_{2}-x_{1}}{y_{2}-y_{1}}\right)$. The resulting baseline length of a minimum of $3.8 \mathrm{~m}$ is not feasible for automotive applications. By using a Laser Tracker instead of a total station, the accuracy could be increased significantly. The Leica absolute tracker AT960 for example has a specified measurement performance well below $1 \mathrm{~mm}$ [6]. With the help of a built-in camera it is possible to determine the 6 DoF pose of a specific probe (T-Mac). Yet, Laser Trackers - typically used in manufacturing - are dependent on stable environmental conditions (vibration, air temperature, air pressure) and have a limited measuring range, usually less than $50 \mathrm{~m}$. In addition, a cable connection between the Laser Tracker and T-Mac is required in most cases and the automated target recognition is not reliable in sunlight. These constraints make this instrument unusable for the task at hand. 
Radar-based systems use microwaves with wavelengths between $1 \mathrm{~mm}$ and $1 \mathrm{~cm}$. A distance measurement with radar can be determined over the time of flight. Radar systems known for navigational purposes do not achieve the high accuracy requirements set for this paper [7]. To carry out high-precision surveying work- typically for monitoring of rockslides or dams - changes in distance are perceived with interferometry. The static setup of radars does not allow an object to be tracked in a partly obstructed environment, and is more useful for surveying an area, not a single moving target. So-called laser-radar systems [8] are used in industrial metrology for surface inspection. However, they are not able to track an object and are thus not suitable for our task.

The iGPS (indoor GPS) technology from 7D Kinematic Metrology (earlier Nikon Metrology) is a local positioning system consisting of several stationary infrared transmitters that emit two inclined beams in a rotating motion. From the time differences of the detected light signals on a sensor, it is possible to calculate the azimuth and the elevation to the transmitter. By measuring the directions to multiple transmitters, it is feasible to calculate a position. In the accuracy assessment of [9], a tracking of velocities up to $3 \mathrm{~m} / \mathrm{s}$ with deviations up to $1.3 \mathrm{~mm}$ can be achieved. The time offset must be considered for spatiotemporal applications, which varies with the speed of the sensor. The systems latency is around $300 \mathrm{~ms}$ [10], and the measurements are not accessible for real-time streaming.

Motion capture Systems estimate the position of retroreflective markers using a stereo setting of active cameras surveying the measurement volume at high frequencies e.g. up to $420 \mathrm{~Hz}$ for a Vicon Vantage V5. The cameras are sensitive to specific wavelengths that either come from retroreflective markers reflecting illuminations from the cameras, or from active luminescent markers. Such motion capture systems are mainly used for movement analysis in movie productions, sports, medical and robotic applications [11].

The positioning performance study of [12] investigates a system with eight Vicon T40S cameras monitoring a rotating arm from distances of $<5 \mathrm{~m}$. His results show high accuracies with errors that range in terms of sub-millimeters. This application can be problematic due to the high number of cameras required for bigger measurement volumes, as well as possible disturbing signals that are caused by sunlight reflections on surfaces.

Light Detection and Ranging (LiDAR) sensors scan the surrounding area at high frequencies and create continuously updated 3D point clouds. With simultaneous localization and mapping (SLAM) methods, a self-localization of the vehicle can be performed. Specifications from LiDAR manufacturers indicate measurement accuracies from $2 \mathrm{~cm}$ and up [13]. Various studies from [14-17] show that with this technique, it is possible to reach root mean square error (RMSE) in the range of decimeters for position measurements, and RMSE of $0.1^{\circ}$ for heading measurement. The results primarily depend on the precision and density of the available 3D data. The computationally intensive algorithms make it hard to implement this technique for real-time applications with high accuracy requirements. In addition, this technology is identical to one of the vehicle sensors that are being tested, which would hinder an independent assessment.

An inertial measurement unit (IMU) with built-in accelerometers and gyroscopes measures the linear accelerations and rotation rates. To derive changes in position, double integration over time is necessary. This measurement principle returns data at high rates from $100 \mathrm{~Hz}$ up to $\mathrm{kHz}[18,19]$, but leads to significant drifts. Therefore, an IMU could complement other measurement systems with high frequent measurements but needs correction values for controlling the drifts.

\section{Materials and Methods}

The proposed tracking system consists of a novel combination of motion capture cameras (Vicon Vantage V5) and robotic MultiStations (Leica MS60).

Instead of the classical motion capture setting - monitoring the motions of markertagged objects from multiple angles with static cameras - the principle is inverted and the cameras are placed in a panoramic view on the moving object (Figure 1), to detect static markers distributed over the area. 


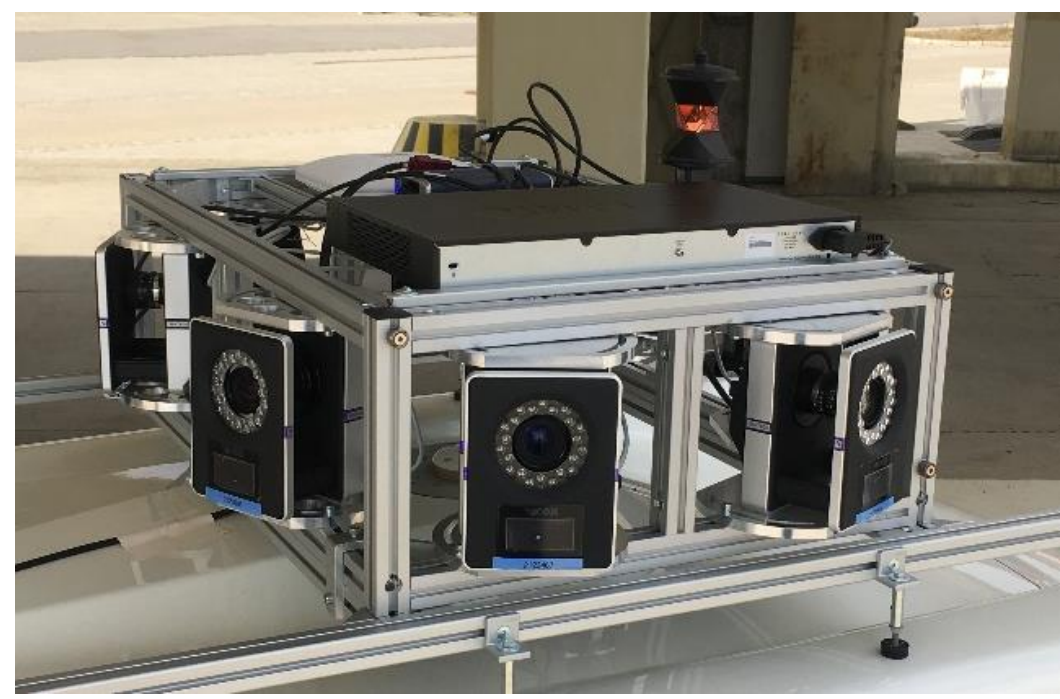

Figure 1. Camera rig with 8 Vicon Vantage V5 cameras and one $360^{\circ}$ prism.

The accuracy of the pose estimation using this panoramic setup is strongly dependent on the distribution and number of markers. With a Vicon Vantage V5 camera, a 1-pixel shift at a $20 \mathrm{~m}$ distance of the marker results in a deviation of $8 \mathrm{~mm}$ respective of $0.023^{\circ}$ in the measured orientation of the camera. With the measurement of a minimum of 3 markers, the 6 DoF pose can be determined. By measuring more markers, the overdetermination allows for an adjustment and outliers can be detected.

The setup of these sensors brings the following advantages to the system:

The motion capture cameras come with integrated pre-processed frames and reveal the image coordinates of detected markers at a stable frame rate of up to $420 \mathrm{~Hz}$ in Standard Mode [20]. Their well-defined shutter time and ability to simultaneously trigger multiple cameras through a master camera enables an accurate synchronization [21]. Installing the cameras onto a mountable rig on top of the car allows the system to be used on different vehicles. The number of cameras used is not dependent on the size of the test site and the energy for the cameras is supplied by the car.

The cheap and light retroreflective markers at the test site can be easily installed on existing infrastructure such as pillars and walls, without any additional construction. Expanding or enhancing a potential test area is fast, and the markers are resistant to environmental influences over longer periods of time.

Total stations provide reliable position information by tracking the position of a $360^{\circ}$ prism on the camera rig during the initialization phase and the maneuvers. Measuring with multiple total stations simultaneously can reduce any systematic errors and allow to monitor more complex scenes with partially obstructed areas.

In addition, the robotic total stations can be used during the setup phase to create a reference frame, measure the coordinates of the retroreflective markers, and acquire positions of additional objects and point clouds. They can also be used to perform the extrinsic calibration between the cameras, the prism and the reference points on the car.

\subsection{Physical Setup}

The system can be divided into stationary components and those placed on the moving car (Figure 2, Figure 3 used in the tracking system. 


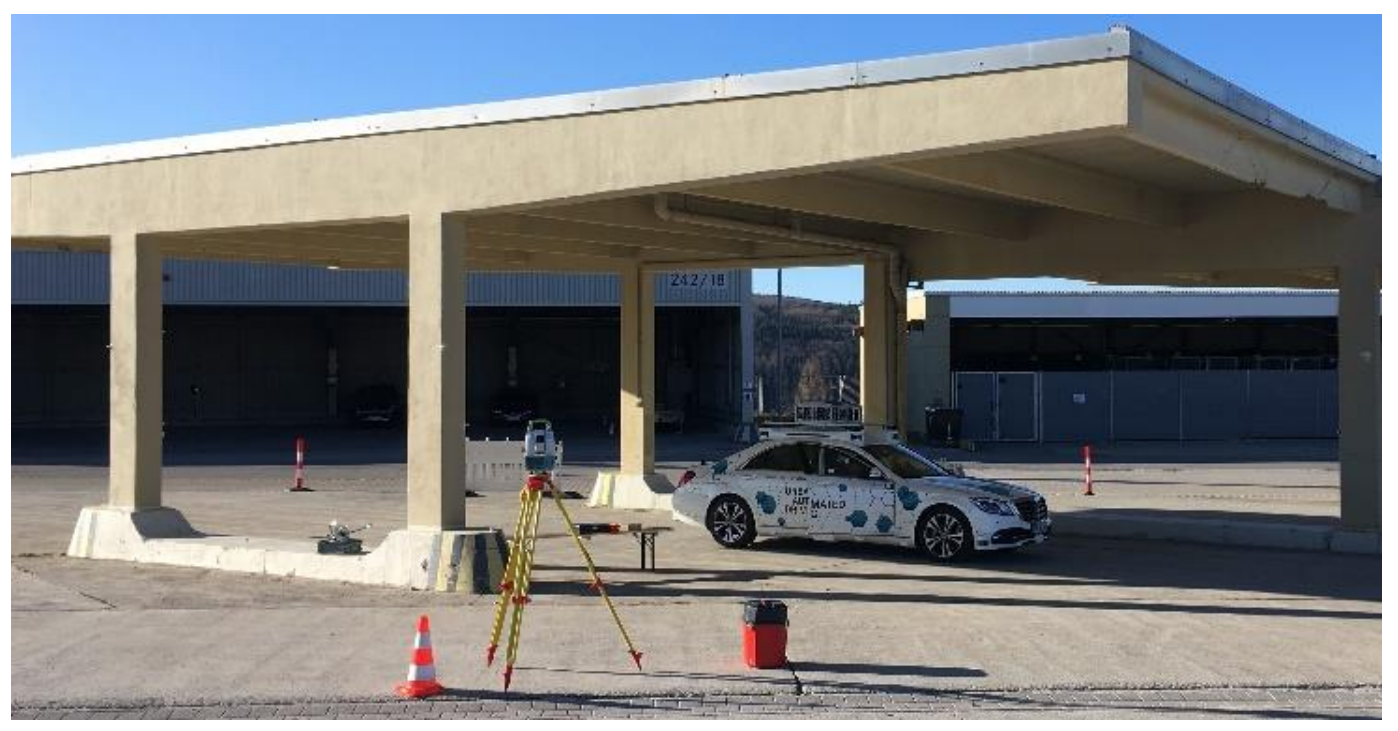

Figure 2. Setup of the tracking system at the test site, consisting of one MS60 (on a tripod in the front) and a camera rig on the roof of the car.

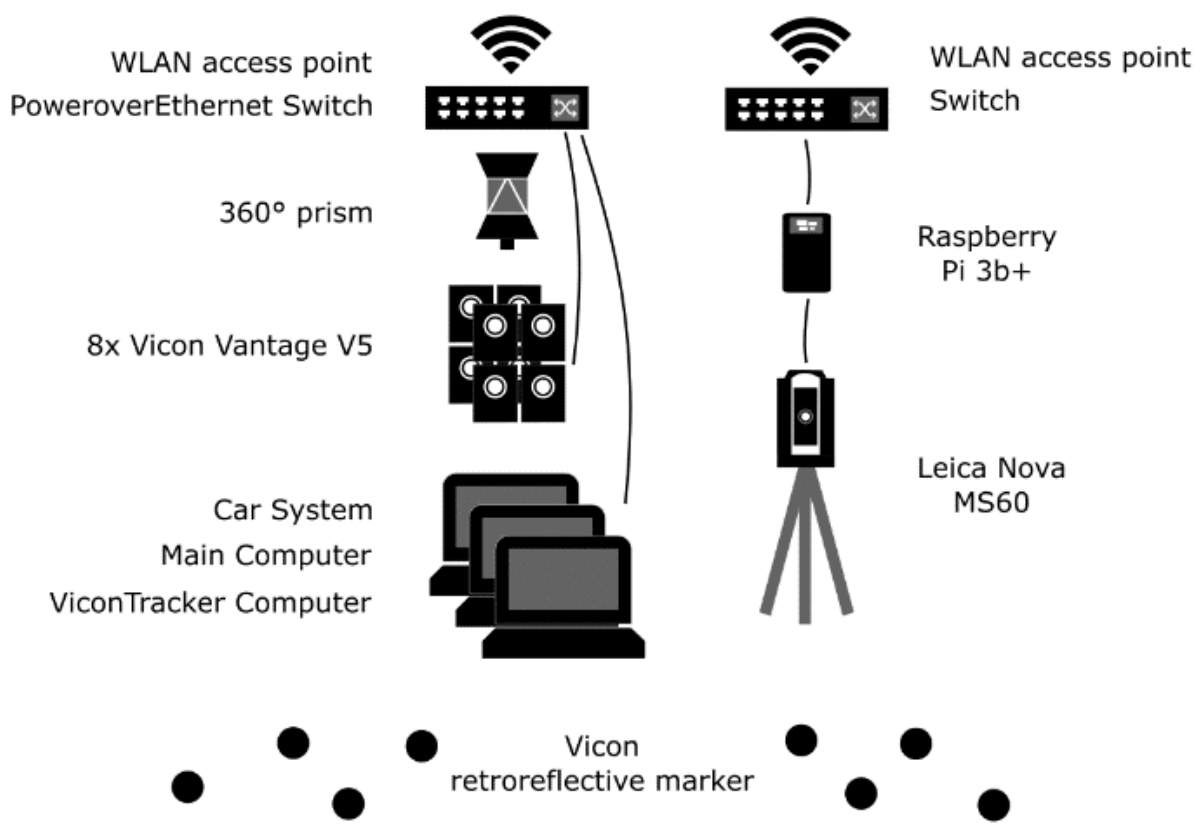

Figure 3. Drawing of the components used in the tracking system.

A (local) world-fixed coordinate frame is established in a first step, along with measurements of the retroreflective markers. The retroreflective markers can automatically be measured by the Leica MS60 ATRplus, up to a range of $15 \mathrm{~m}-25 \mathrm{~m}$ depending on the lighting conditions. To calculate the correct distances, a new prism type must be defined in the total station with an absolute constant of $19.0 \mathrm{~mm}$. To improve accuracy and reliability, the markers are measured additionally from a second total station setup.

In order to exchange messages with the network on the car, the battery powered MS60 are connected via TCP/IP to a USB port on a Raspberry Pi 3b+, that is then connected via an Ethernet cable to a switch with a WLAN access point.

All system components placed on the car (except the computers) are attached to a rig (Figure 1). This includes a bolt for mounting the $360^{\circ}$ prism, the complementing WLAN access point, and a switch that gets its power from the car and supplies the motion capture cameras with Power over Ethernet (PoE).With eight Vicon Vantage 5 cameras, it is possible to achieve a coverage of $95 \%$ of the horizon at a distance of $5 \mathrm{~m}$ from the car. 
This rig forms a coordinate system whose origin is the optical center of the prism, while the definition of a main camera determines the orientation. All cameras (independently and intrinsically calibrated beforehand) as well as the prism are calibrated to each other, so that the system can determine the pose of the rig in relation to the worldfixed coordinate system.

By measuring reference points on the car, a static transformation from the rig to the car-fixed coordinate system is established.

The connection to the vehicle's autonomous system is established over the PoE switch and Ethernet cables, along with the computers needed for controlling the cameras and processing the data.

\subsection{Logical Setup}

A Precision Time Protocol (PTP) grandmaster clock provided by the car's system serves as basis for the synchronization. The results section describes the procedures that implement an accurate timestamp of the measurements to the actual sensor recording time, as well as an analysis of the obtained accuracy.

The communication between the components is done via ROS nodes, and the master is provided by the car's system.

ROS nodes can be used to subscribe and publish topics, as well as process the data. All of the published messages include timestamps and receiving a message from a subscribed topic can be used as a callback to trigger functions. Another method that is used are loops which are triggered at a pre-defined rate (or slower if the calculations take longer). In addition, the package tf2 [22] is used to keep track of the different coordinate frames as well as their transformations to each other, which provides buffered information up to 10 seconds into the past. Figure 4 . shows the information flow that is now explained in detail.

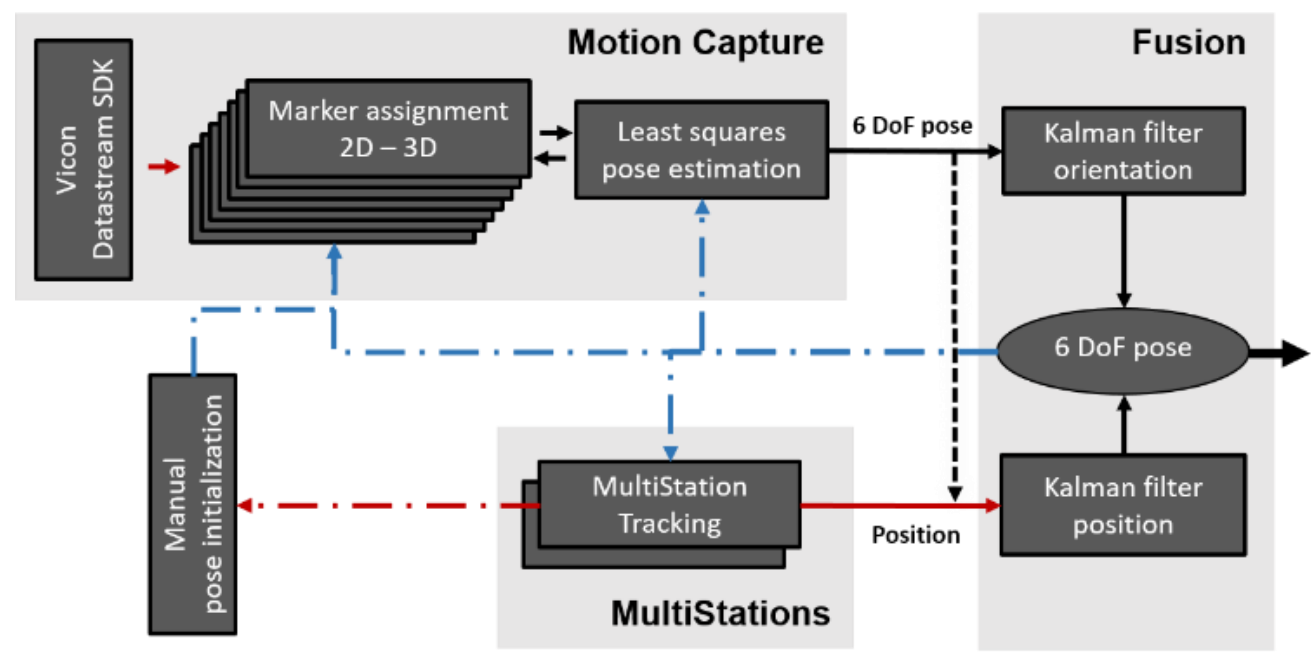

Figure 4. The core elements of the logical implementation. Each box represents one ROS-node. The measurements are in red, and the blue dash-dotted arrows are pose messages that are needed as initial values.

A script running on Raspberry Pi triggers the coordinate measurements of the prism at approximately $22 \mathrm{~Hz}$, using the MS60 via the Leica GeoCOM interface. Measurement data is then transmitted back to the Raspberry Pi via the same interface. Based on the measured prism position provided by a total station, other total stations in the network are actively aimed towards the prism's position to enable a fast and automatic lock when the prism enters their field of view.

The measurements - coordinates and radii of the markers - provided by the cameras are read out from the Vicon Datastream SDK and are then published as messages into a ROS topic at a rate of $80 \mathrm{~Hz}$. 
On system startup, the MS60s deliver the prism's position information. With a manually estimated heading the orientation of the rig is initialized by visually aligning the superimposed projected markers to the camera images. After the initialization, the markers are tracked in the image space by each camera simultaneously. The markers that were previously matched are then explored within a predefined search window. For any undetected or unmatched point, a new match is searched for in the image space - using the last published pose for the projection.

For scenarios with obstructed views, areas of visibility are assigned to the markers so that they are only used for matching if they are visible at the moment, to avoid any wrong assignments.

With all the matches of all the cameras, a least square estimation for the pose is calculated based on the following formula

$$
K_{C_{i}}^{-1} * x=\left[R_{b}^{C_{i}} * R_{w}^{b} \mid-R_{b}^{C_{i}} * R_{w}^{b} *\left(r_{w, b}^{w}+R_{w}^{b^{T}} * r_{b, C_{i}}^{b}\right)\right] * X
$$

$X, x$ coordinates in $3 \mathrm{D} / 2 \mathrm{D}$

$K \quad$ camera matrix

$R \quad$ rotation matrices - start system (bottom) to target system (top)

$r \quad$ calibrated vector between two points

$w=$ world $b=$ base link, $C i=\mathrm{i}$-th camera

This algorithm uses iterations that converge to the optimal solution, which depends on approximate initial values. These iterations form the bottleneck for near-real-time processing. Therefore, only every second timeframe from the marker tracker is used for the pose estimation $(=40 \mathrm{~Hz})$, and the maximum number of iterations is set to three, irrespective of whether the convergence threshold is met. Markers with residuals above a preset threshold are declared as outliers, and their matching confidence values are reduced for the following estimations.

Two independent Kalman filters are used to achieve more robust position and orientation determinations. Both are set to run at a rate of $25 \mathrm{~Hz}$. The Kalman filter for the position fuses the measurements from multiple MS60s and, optionally, from the calculated Vicon pose (dashed line Figure 4). In contrast, for the orientation, the only source of measurement is the pose estimation. For the orientation, the Kalman filter helps detect wrongly estimated poses and overcomes short periods of missing pose estimations.

The components of both Kalman filters are combined into the final pose, which can, in turn, be used as an approximate pose for all other nodes.

Errors introduced by the latency of pose messages are reduced by extrapolating from the short-term pose history to the current timestamp.

\subsection{Visualization and Post-processing}

The ROS topics can be recorded into rosbags for post-processing purposes such as replaying recordings, reprocessing the data, or doing additional evaluations such as distance measurement comparisons between the optical tracking system and the sensors on the vehicle. Monitoring the system status while recording is also possible using a realtime visualization in RViz, which shows the car's movements (

Figure 5) and status messages concerning the different subsystems. 


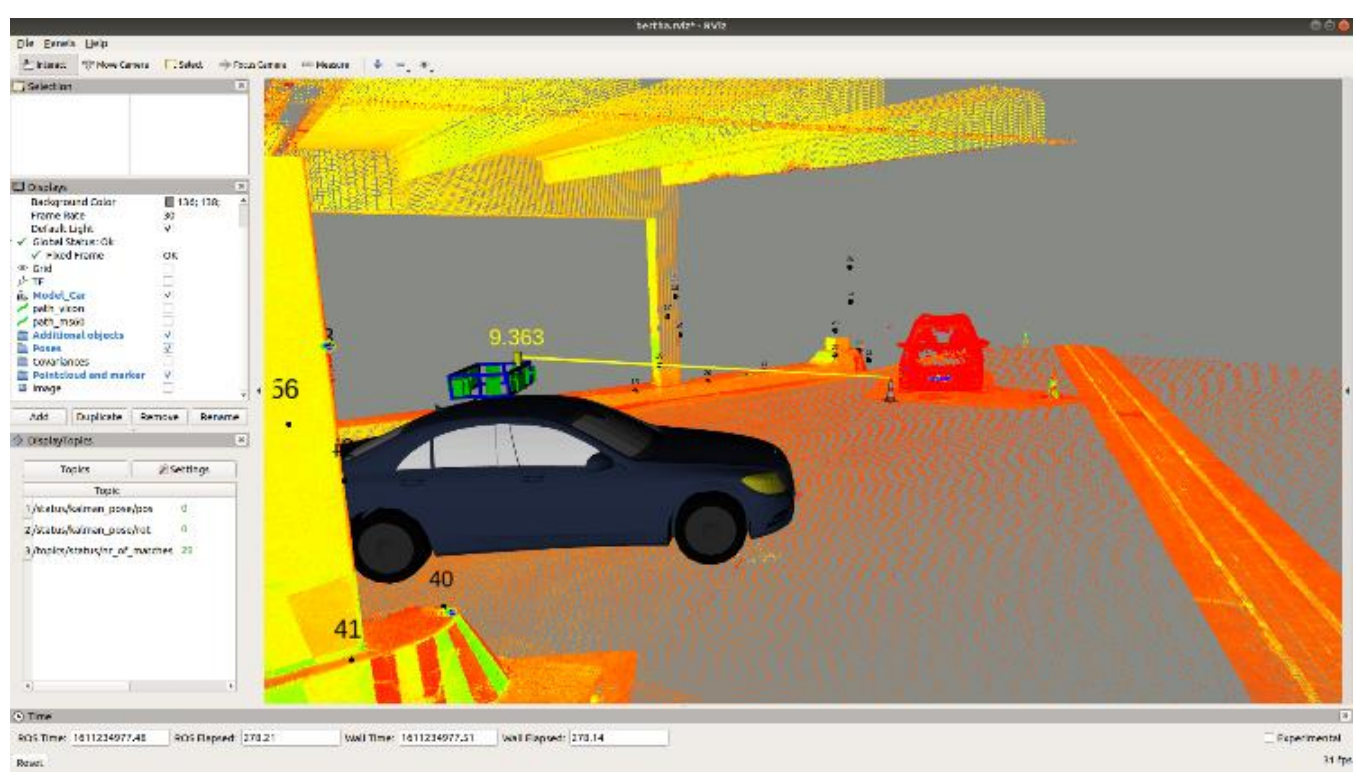

Figure 5. Visualization of the point cloud, which represents the static digital twin of the test scene and the moving digital twin of the car.

\section{Results}

\subsection{Requirements Coverage}

The implemented system along with the combination of Leica MS60 MultiStations and Vicon motion capture cameras allows us to achieve all the requirements needed for the industrial application as shown in Table 1.

Table 1. List of requirements.

\begin{tabular}{cccccc}
\hline Requirement & Target & $\begin{array}{c}\text { Achieved } \\
\text { overall }\end{array}$ & $\begin{array}{c}\text { Achieved by } \\
\text { MS60 }\end{array}$ & $\begin{array}{c}\text { Achieved by } \\
\text { Vicon }\end{array}$ & Requirement \\
\hline Frequency & $>20 \mathrm{~Hz}$ & $25 \mathrm{~Hz}$ & $20 \mathrm{~Hz}$ & $40 \mathrm{~Hz}$ & Frequency \\
Latency & $500 \mathrm{~ms}$ & $200 \mathrm{~ms}$ & $50-60 \mathrm{~ms}$ & $40 \mathrm{~ms}$ & Latency \\
$\begin{array}{c}\text { Positional error } \\
\begin{array}{c}\text { Orientational } \\
\text { error }\end{array}\end{array}$ & $5 \mathrm{~mm}(3 \sigma)$ & $5 \mathrm{~mm}$ & $5 \mathrm{~mm}$ & $5-30 \mathrm{~mm}$ & $\begin{array}{c}\text { Positional error } \\
\text { Orientational } \\
\text { error }\end{array}$ \\
\hline
\end{tabular}

In addition to these numerical requirements, a synchronization using PTP and full control of the system from within the test vehicle is achieved.

\subsection{Synchronization}

A good synchronization of all the sensors creates a basis for accurate spatio-temporal data. The required accuracy of the synchronization depends on the maximal velocity of the object and the targeted position accuracy as shown in Table 2.

Table 2. Relations between velocity, synchronization and accuracy.

\begin{tabular}{ccc}
\hline Velocity & $\begin{array}{c}\text { Targeted position } \\
\text { accuracy @ 10 }\end{array}$ & $\begin{array}{c}\text { Requirement of } \\
\text { maximal synchroni- } \\
\text { zation offset }\end{array}$ \\
\hline $1 \mathrm{~m} / \mathrm{s}$ & $1 \mathrm{~mm}$ & $1 \mathrm{~ms}$ \\
$1 \mathrm{~m} / \mathrm{s}$ & $5 \mathrm{~mm}$ & $5 \mathrm{~ms}$ \\
$5 \mathrm{~m} / \mathrm{s}$ & $5 \mathrm{~mm}$ & $1 \mathrm{~ms}$ \\
\hline
\end{tabular}


The internal synchronization of the car is established via a grandmaster clock that communicates over PTP. The same grandmaster clock is used for synchronizing the optical tracking system computers in software stamping mode. The following sections describe the additional procedures needed to synchronize the sensors' time systems to PTP.

The motion capture data streamed from the Datastream SDK show a latency of around $40 \mathrm{~ms}$. For the camera synchronization, the UDP trigger signals sent from the main camera are used to connect the frame numbers of the motion capture System to the PTP timestamp. An additional offset dependent on the frame rate is applied, in order to consider the offset between the UDP trigger signal and the actual shutter opening time. With the frame rate of $80 \mathrm{~Hz}$, it corresponds to $11.5 \mathrm{~ms}$.

For the MS60, the PTP synchronization over WLAN between the RaspberryPi slave (using the PTP implementation ptpd2) and the grandmaster clock is investigated. Over a period of multiple hours, the analysis shows a mean offset from the master clock of $0.01 \mathrm{~ms}$ with a $3 \sigma$ standard deviation of $1 \mathrm{~ms}$ (Figure 6).

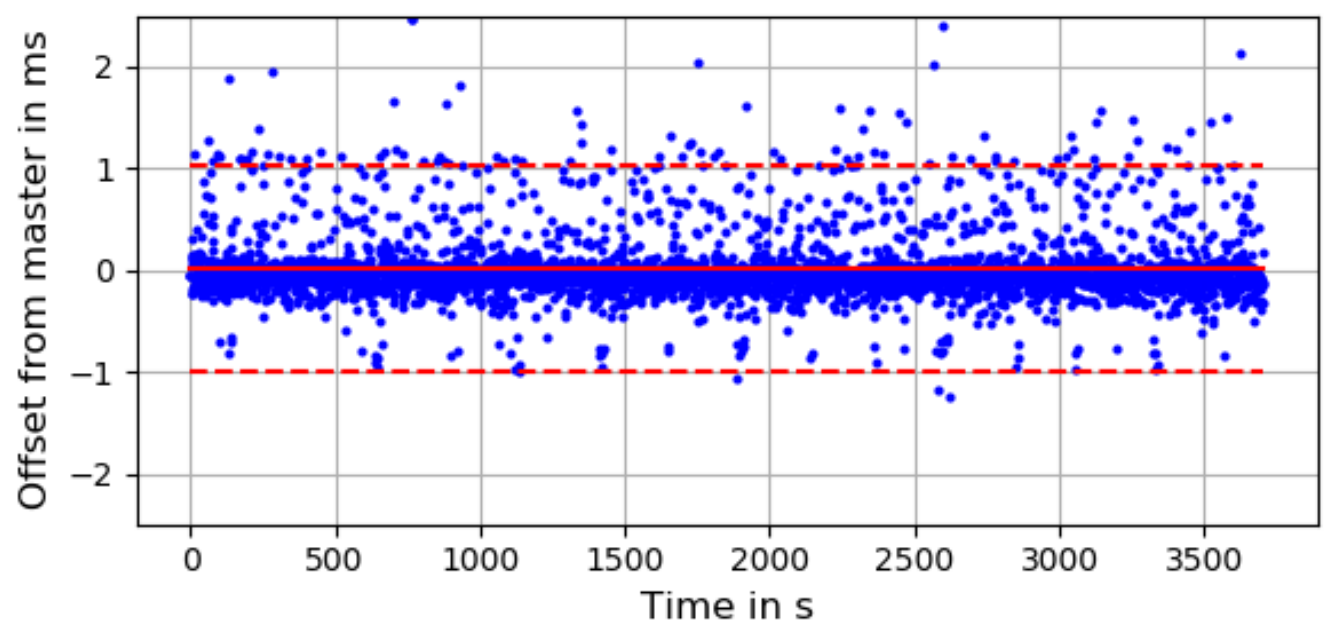

Figure 6. Time offsets of a slave to its master over WLAN.

The link between the timestamps as delivered by the MS60s' measurements to the PTP time is established through the estimation of a clock model with a scale and offset:

$$
t_{P T P}=t_{M S} * m+t_{0}+t_{c}
$$

$t_{P T P}$ ROS-time

$t_{M S}$ MS60-time

$m$ time scale

$t_{0} \quad$ offset

$t_{c} \quad$ constant offset for numerical reasons

To query the MS60 sensor time, a dedicated GeoCOM command that takes approximately $10 \mathrm{~ms}$ between request and receiving - typical values for other requests have a duration above $40 \mathrm{~ms}$ - delivers the observations for calculating the time offset of the MS60. Assumingly, the returned time is taken from the middle third of the entire timespan, so an unknown offset of 3.3 ms remains.

At startup, the clock model is initialized with 180 samples of the sensor time. During the tracking of the prism, a new sample is taken every three seconds that is weighted dependent on the time span between sending and receiving. Every 30 seconds, the parameters $m$ and $t_{0}$ are newly estimated. Investigations show that these sparse updates are more than sufficient, and even after 10 minutes without an update, no significant drift of the clock model appears. 
The reliability of the continuous synchronization is controlled by measuring independent samples every five seconds and comparing their deviations to the clock model. The results of two hours of measurements show a mean shift of -0.03 and a $3 \sigma$ standard deviation of $1.02 \mathrm{~ms}$.

In summary, the synchronization of the MS60 has an empirically determined standard deviation $(3 \sigma)$ of $1 \mathrm{~ms}$ due to the WLAN usage and $1.02 \mathrm{~ms}$ due to a random scatter. Due to the unknown measuring time an unknown offset - estimated to be in the range of $3.3 \mathrm{~ms}$ - remains.

\subsection{Position Accuracy MS60}

Measurements from two MS60 onto one moving prism are used to quantify the velocity-dependent influence of the synchronization and the automatic target recognition (ATRplus) on the coordinate accuracy. Other potential error sources were reduced by positioning the MS60 close to each other - resulting in a minimal influence of the orientation of the $360^{\circ}$ prism - and using the same fixpoints for the resection. The measured trajectories were smooth movements over an area of $15 \times 50 \mathrm{~m}$, with measuring distances up to 50 $\mathrm{m}$ tracked at a rate of $20 \mathrm{~Hz}$.

Table 3 shows the comparison between the two trajectories, where for each measurement of one MS60, the corresponding coordinates of the second trajectory are determined via a linear interpolation over time.

These results are influenced by the errors occurring on both MS60 and show the expected results of increased differences for higher velocities. For these higher velocities, some of the deviations are caused by the linear interpolation of a curved trajectory.

Table 3. Coordinate differences dependent on the velocity.

\begin{tabular}{ccccc}
\hline Velocity & $\begin{array}{c}\text { Mean of coordi- } \\
\text { nate differences in } \\
\text { mm }\end{array}$ & $\begin{array}{c}\text { Standard deviation } \\
\text { of single measure in } \\
\text { mm }\end{array}$ & $\begin{array}{c}\text { Maximal } \\
\text { difference in } \\
\text { mm }\end{array}$ & samples \\
\hline $0 \mathrm{~m} / \mathrm{s}-1 \mathrm{~m} / \mathrm{s}$ & 2.0 & 0.4 & 4.5 & 2281 \\
$1 \mathrm{~m} / \mathrm{s}-2 \mathrm{~m} / \mathrm{s}$ & 4.2 & 3.9 & 22.2 & 1782 \\
$2 \mathrm{~m} / \mathrm{s}-3 \mathrm{~m} / \mathrm{s}$ & 7.2 & 5.5 & 44.7 & 2062 \\
$3 \mathrm{~m} / \mathrm{s}-4 \mathrm{~m} / \mathrm{s}$ & 8.9 & 7.7 & 55.4 & 781 \\
$4 \mathrm{~m} / \mathrm{s}-5 \mathrm{~m} / \mathrm{s}$ & 8.8 & 7.4 & 64.1 & 620 \\
\hline
\end{tabular}

These results show that the tracking functionality of MS60 only meets the requirements at slower speeds - as they appear in the proposed application area with near-range maneuvers - and that the standard deviation of the differences is very small compared to the mean value.

\subsection{Comparison of Position Accuracy of Subsystems}

The measured position of the system's reference point located at the prism center can be determined independently by multiple MS60 and from the 6DoF pose estimation from the motion capture System. A comparison of these positions can be used to check single measurements and to detect continuous offsets caused by erroneous setups or calibrations.

Figure 7 shows a seven second excerpt of a maneuver performed in the laboratory on a moving table. In Figure 8, a more detailed view of the same maneuver in the encircled region is shown. It indicates a small spike from the motion capture pose estimation that can be cleaned up in a post-processing step, as well as a dynamic offset between the two MS60 that can be explained through cyclic deviations introduced by the $360^{\circ}$ prism. 


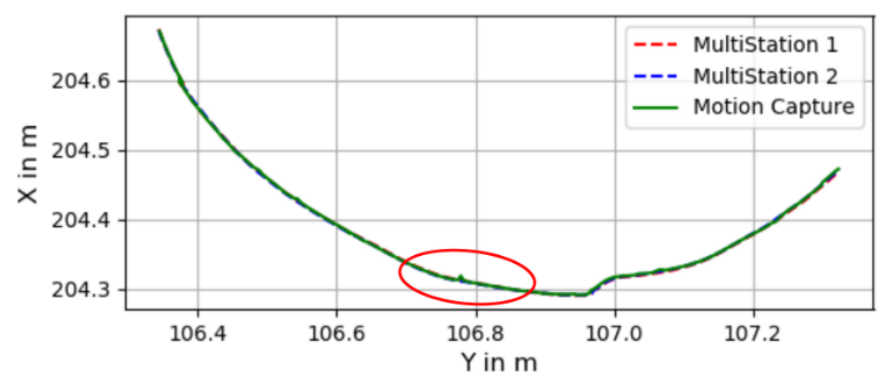

Figure 7. Measurements of a circular rotation.

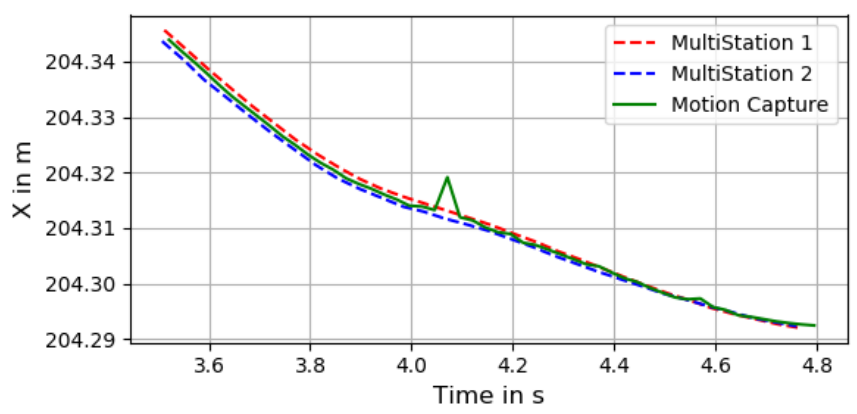

Figure 8. Detailed view of the encircled region in Figure 7.

\subsection{Orientation Accuracy Motion Capture System}

The resulting orientations of the pose estimations are checked with a laser tracker AT401 that has a 3D point accuracy in the range of $+/-15 \mu \mathrm{m}+6 \mu \mathrm{m}$.

For these checks, the system is set up in five different poses within a laboratory environment with 20 - 25 retroreflective markers in the motion capture camera's field of view. Three points on the rig that form the basis of a coordinate system are measured by the laser tracker (

Figure 9) to determine the orientation of the pose independently.

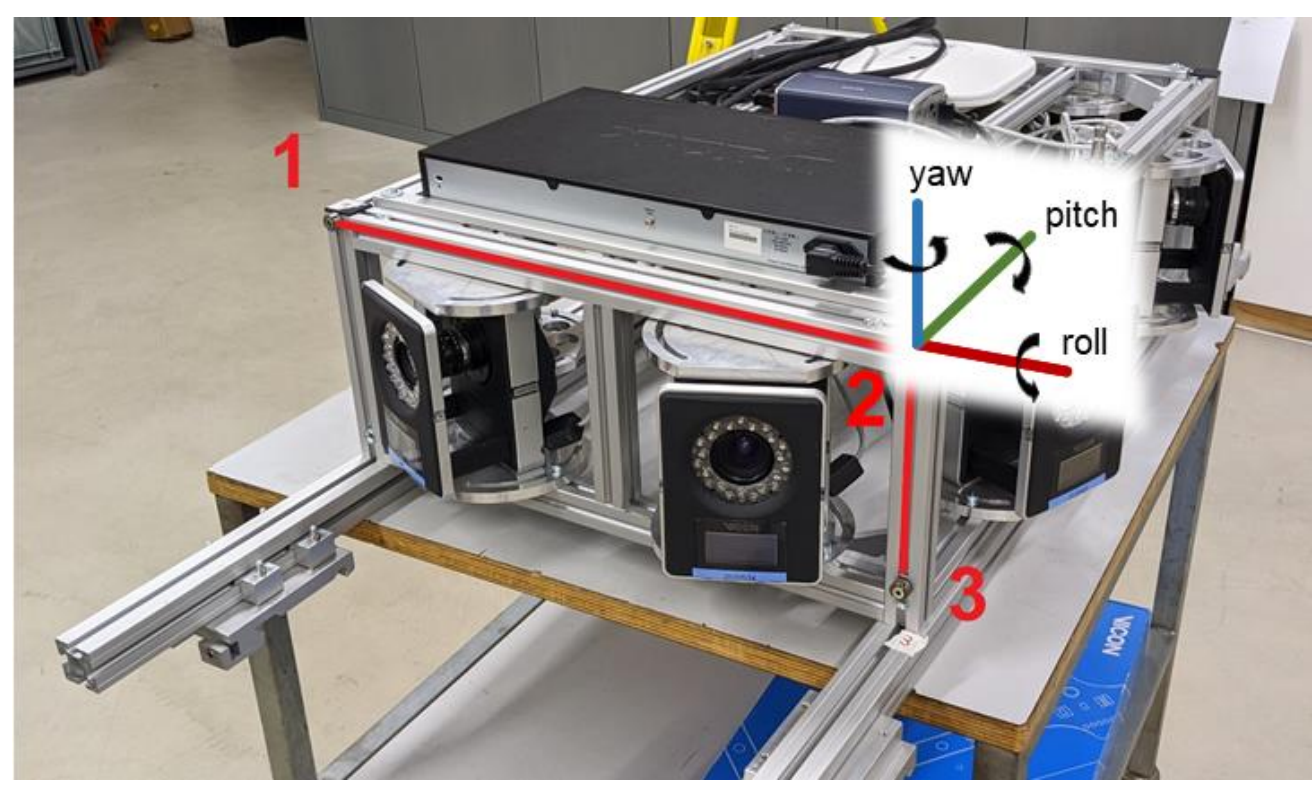

Figure 9. Reference points for the laser tracker measurements.

As listed in Table 4, the resulting standard deviation of these static measurements shows a clear achievement of the requirements under these optimal conditions.

Table 4. Resulting standard deviations around the axes. 


\begin{tabular}{cccc}
\hline & roll in deg & $\begin{array}{c}\text { pitch in } \\
\text { deg }\end{array}$ & $\begin{array}{c}\text { yaw in } \\
\text { deg }\end{array}$ \\
\hline $\begin{array}{c}\text { Standard } \\
\text { deviation } \\
(1 \sigma)\end{array}$ & 0.0215 & 0.0255 & 0.0133 \\
\hline
\end{tabular}

The smaller standard deviations around the z-axis can be explained through the configuration of the cameras distributed in a panoramic view around that axis, whereas the reliability around the other axes is less strongly controlled.

\section{Discussion}

The proposed optical tracking system provides an integrated workflow to prepare a digital twin of the test scene. It also uses geodetic total stations and the motion capture System to determine a highly accurate $6 \mathrm{DoF}$ pose estimation as a reference for the validation of an autonomous driving vehicle in near-range scenarios. The system can cover a test-drive distance up to $100 \mathrm{~m}$, which is sufficient for most parking scenarios and environments.

To achieve the given requirements, every step must be performed at the highest level of accuracy. This includes establishing an accurate and stable fixpoint network, the distribution and measurement of the retroreflective markers, the relative calibration of all sensors, as well as the synchronization of all sensors.

By using the optical tracking system, a vehicle's sensory and algorithmic output can be evaluated in real-time during the execution of near-range maneuvers. This is achieved by visualizing the algorithmic output and the car's reference pose on the onboard display unit, or on any laptop connected to the vehicle. In addition, the data can be recorded in rosbag files for later in-depth investigation and validation of the autonomous vehicle.

To further improve the optical tracking system, the following options could be considered:

- An improvement of the MS60s' measurements could be achieved through enhanced synchronization. However, this would require changes to the MS60s' firmware or even the hardware.

- $\quad$ As suggested by [23], an application of corrections mitigating the systematic cyclic deviations of the $360^{\circ}$ prism can be pursued, since the alignment of the prism towards the MS60 is always known.

- In addition, supporting the orientation determination through the integration of an IMU into the existing system could make the marker detection a more robust assignment.

Author Contributions: Conceptualization, Ursula Kälin, Louis Staffa and David Grimm; Data curation, Ursula Kälin, Louis Staffa and David Grimm; Formal analysis, Ursula Kälin and David Grimm; Funding acquisition, Axel Wendt; Investigation, Ursula Kälin; Methodology, Ursula Kälin and David Grimm; Project administration, Louis Staffa; Resources, Louis Staffa and Axel Wendt; Software, Ursula Kälin; Supervision, David Grimm; Validation, Ursula Kälin, Louis Staffa, David Grimm and Axel Wendt; Visualization, Ursula Kälin; Writing - original draft, Ursula Kälin, Louis Staffa, David Grimm and Axel Wendt; Writing - review \& editing, David Grimm.

All authors have read and agreed to the published version of the manuscript.

Funding: This research received no external funding.

Data Availability Statement: Data sharing is not applicable to this article.

Conflicts of Interest: The authors declare no conflict of interest.

\section{References}

1. Leica Geosystems AG. Leica Nova MS60 Data Sheet 2015.

2. Lackner, S.; Lienhart, W. Impact of Prism Type and Prism Orientation on the Accuracy of Automated Total Station Measurements.; 2016. 
3. Lienhart, W.; Ehrhart, M.; Grick, M. High Frequent Total Station Measurements for the Monitoring of Bridge Vibrations. Journal of Applied Geodesy 2017, 11, 1-8, doi:10.1515/jag-2016-0028.

4. Stempfhuber, W. Synchronisation von Zielverfolgenden Servotachymetern Für Kinematische Anwendungen. Kinematische Messmethoden - Vermessung in Bewegung, Schriftenreihe des DVW 2004, 45, 151-163.

5. Thalmann, T.; Neuner, H. Untersuchung Des Network Time Protocols Für Die Synchronisation von Multi-Sensor-Systemen. avn-allgemeine vermessungsnachrichten 2018, 125, 163 - 174.

6. Hexagon Manufacturing Intelligence. LEICA ABSOLUTE TRACKER AT960 ASME B89.4.19-2006 Specifications 2016.

7. AutonomouStuff. Radar Comparison Chart. Available online: https://autonomoustuff.com/radar-chart (accessed on 1 February 2021).

8. Nikon. Laser Radar MV331/351 Automated Large Volume Inspection 2020.

9. Depenthal, C. Path Tracking with IGPS. In Proceedings of the 2010 International Conference on Indoor Positioning and Indoor Navigation; IEEE: Zurich, Switzerland, 2010; pp. 1-6.

10. Norman, A.R.; Schönberg, A.; Gorlach, I.A.; Schmitt, R. Validation of IGPS as an External Measurement System for Cooperative Robot Positioning. The International Journal of Advanced Manufacturing Technology 2013, 64, 427-446, doi:10.1007/s00170-012-40048.

11. Vicon. TheStandard 2020 Edition 2020.

12. Merriaux, P.; Dupuis, Y.; Boutteau, R.; Vasseur, P.; Savatier, X. A Study of Vicon System Positioning Performance. Sensors 2017, 17, 1591, doi:10.3390/s17071591.

13. AutonomouStuff. LiDAR Comparison Chart. Available online: https://autonomoustuff.com/lidar-chart (accessed on 1 February 2021).

14. Wolcott, R.W.; Eustice, R.M. Visual Localization within LIDAR Maps for Automated Urban Driving. In Proceedings of the 2014 IEEE/RSJ International Conference on Intelligent Robots and Systems; IEEE: Chicago, IL, USA, 2014; pp. 176-183.

15. Wang, L.; Zhang, Y.; Wang, J. Map-Based Localization Method for Autonomous Vehicles Using 3D-LIDAR. IFAC-PapersOnLine 2017, 50, 276-281, doi:10.1016/j.ifacol.2017.08.046.

16. Javanmardi, E.; Javanmardi, M.; Gu, Y.; Kamijo, S. Autonomous Vehicle Self-Localization Based on Multilayer 2D Vector Map and Multi-Channel LiDAR. In Proceedings of the 2017 IEEE Intelligent Vehicles Symposium (IV); IEEE: Los Angeles, CA, USA, 2017; pp. 437-442.

17. Sobreira, H.; Costa, C.M.; Sousa, I.; Rocha, L.; Lima, J.; Farias, P.C.M.A.; Costa, P.; Moreira, A.P. Map-Matching Algorithms for Robot Self-Localization: A Comparison Between Perfect Match, Iterative Closest Point and Normal Distributions Transform. Journal of Intelligent $\mathcal{E}$ Robotic Systems 2019, 93, 533-546, doi:10.1007/s10846-017-0765-5.

18. Novatel Inc. SPAN UIMU-LCI. Data Sheet. Available online: http://www.seabed.nl/wp-content/uploads/ 2015/12/IMU-LCI.pdf (accessed on 25 May 2018).

19. Xsens. MTi 100-Series. Data Sheet. Available online: https://www.xsens.com/download/pdf/documentation/ mti-100/mti-100series.pdf (accessed on 25 May 2018).

20. Vicon. Vicon Vantage Reference Guide 2020.

21. Stucki, P. Synchronisierung Eines Vicon Motion Capture Systems Für Ki-Nematische Anwendungen Mit ROS. Presented at the Frontiers of geodetic science 2019, 2019.

22. Foote, T. Tf: The Transform Library. Presented at the Technologies for Practical Robot Applications (TePRA), 2013 IEEE International Conference On Open-Source Software Workshop, 2013.

23. Favre, C.; Hennes, M. Zum Einfluss der geometrischen Ausrichtung von $360^{\circ}$-Reflektoren bei Messungen mit automatischer Zielerfassung. Vermessung, Photogrammetrie, Kulturtechnik: VPK 2000, 72-78. 